\title{
Article
}

\section{A Binomial Model of Asset and Option Pricing with Heterogeneous Beliefs}

\author{
Xue-Zhong He and Lei Shi *
}

University of Technology Sydney, Business School, Finance Discipline Group, P.O. Box 123 Broadway, NSW 2007, Australia; Tony.He-1@uts.edu.au

* Correspondence: Lei.Shi@uts.edu.au; Tel.: +61-2-9514-7772; Fax: +61-2-9514-7711

Received: 26 August 2016; Accepted: 22 September 2016; Published: 19 October 2016

\begin{abstract}
This paper provides a difference-in-opinions equilibrium framework for pricing asset and option in a multi-period binomial economy with heterogeneous beliefs. Agents agree to disagree about their beliefs on the probability and asset return in each state of nature. By constructing a consensus belief, we examine the impact of heterogeneous beliefs on market equilibrium. We show that agents' wealth shares are expected to remain the same under the consensus belief, although they are expected to increase under their own beliefs. Also large disagreement leads to lower risk premium, while high disagreement on the future return in up state (down state) leads to lower (higher) risk-free rate and expected return for the risky asset. Furthermore, under the consensus belief, the implied volatility of the call options exhibits some observed patterns widely documented in option markets.
\end{abstract}

Keywords: Asset prices, Heterogeneous beliefs, Binomial trees, Options

JEL Codes: G12, D84

\section{Introduction}

The binomial model was first proposed by Cox, Ross and Rubinstein (1979) (CRR). Since then it has been widely used in the literature. At the time of its publication, economists were not conversant with the mathematical tools used to derive the Black-Scholes option pricing formula. The binomial model uses a discrete-time binomial lattice (tree) framework to model the dynamics of the underlying stock price. It can be characterized simply by the probability of an up move and sizes of the move in the up and the down states. When all three parameters are constant, with appropriate specification, the binomial lattice converges weakly to the BlackScholes (BS) model in continuous time limit. ${ }^{1}$ Because of its simplicity, the binomial model provides a simple

\footnotetext{
1 Kloeden and Platen (1992) show that the Euler scheme converges weakly to a diffusion process if one replaces the Wiener increment in the Euler scheme with a two-point distributed random variable. The resulting numerical scheme is a binomial lattice with time and state dependent upward and downward moves, which are equal likely to occur. The parameters can also be set for the binomial lattice to weakly converge to other popular diffusion models used in finance.
} 
framework to model stock price dynamics and interest rate term structure. ${ }^{2}$

Empirically, it is commonly observed that the implied volatility from the Black-Scholes option prices is not constant with respect to either the strike price or the time to maturity, which violates the constant volatility assumption underlying the BS model (see for instance Rubinstein (1985, 1994) and Dumas et al. (1998)). Empirically, it is widely observed that the implied volatilities are downward sloping with respect to moneyness and expirations of the options. Several pricing models have been proposed to overcome these problems, including the stochastic volatility models (Hull and White (1987), Wiggins (1987), Melino and Turnbull (1990), Heston (1993)), GARCH models (Duan (1995), Heston and Nandi (2000)) and models with jumps in the underlying price process (Merton (1976), Bates (1991)). By modifying the stochastic process followed by the underlying asset price, some of these models have been able to calibrate to the observed volatility surface. However, apart from the stability problem of the estimated volatility surface, these models do not provide an economic explanation for the phenomenon observed in option markets. In this paper, we consider a multi-period binomial economy with heterogeneous beliefs and use the differences-in-opinion equilibrium approach to provide such an explanation. In this framework, agents agree to disagree about their beliefs on the probability and asset return in each state of nature. We show that the market equilibrium can be characterized by a consensus belief. In market equilibrium, the wealth shares of all the agents are expected to have no change and the implied volatility of European call options exhibits some of the observed patterns documented in option markets.

This paper follows the equilibrium asset pricing model literature with heterogeneous beliefs developed recently ${ }^{3}$. We consider a simple economy with one risky and one risk-free assets. The stock price follows the CRR model. Agents are log-utility maximizers of their terminal wealth. They have heterogeneous beliefs about the probability and the size of the moves in each state. This means that agents agree to disagree and the differences in opinion are due to the interpretation of the same information. Different from the current literature, we do not impose any exogenous quantity (neither the stock price nor the dividend process) other than the current wealth shares of the agents and their subjective beliefs on the future evolution of the stock price on the binomial lattice. The binomial model is not restrictive in the sense that the probability of an up move and the size of price changes can be both time and state dependent, implying a wide range of stochastic models that can be incorporated into this framework. Since agents have different beliefs about the future stock price in each state, they disagree on the state prices. Therefore they price options differently. The question is, what should be the fair price of the option? To answer this question, we follow Jouini and Napp (2006, 2007) and Chiarella et al. $(2010,2011)$ and construct a consensus belief in market equilibrium. We show that the consensus belief turns out to be a fair belief under which the wealth shares of all the agents are martingale, though agents' expected wealth shares increase under their own beliefs. We then use the consensus belief to price option and show that the implied volatility of the option price exhibits the volatility skewness.

This paper is closely related to the market selection literature that concerns the survival of irrational or noise traders and their impact on the equilibrium price in the long run. This strand of literature was originated from the hypothesis of Friedman (1953) that irrational traders do not survive in the long run and therefore have no price impact in the long run. Under different model setup, DeLong et al. $(1990,1991)$ have found that

\footnotetext{
${ }^{2}$ For example, Nelson and Ramaswamy (1990) develop methods to construct a recombined binomial lattice for diffusion model to enhance computational efficiency. Hahn and Dyer (2008) apply the method to mean-reverting stochastic processes specifically for real option valuation. van der Hoek and Elliott (2006) present a text book treatment for binomial models and their applications. 3 See, for example, Detemple and Murthy (1994), Zapatero (1998), Basak (2000, 2005), Abel (2002), Jouini and Napp (2006, 2007), David (2008), Berraday (2009), Chiarella, Dieci and He (2010, 2011).
} 
noise trading can be persistent. Blume and Easley (2006) show that irrational traders can dominate rational traders in an incomplete market, Sandroni (2000) and Dumas et al. (2009) show that irrational trader becomes extinct only after a long time. Kogan et al. (2006) provide evidence that price impact and survivability are two different concepts, irrational traders can be very close to extinction yet still have a significant price impact. Most of the models assumes that there exists at least one rational agent who knows the true or objective law of motion governing the exogenous process (dividend or endowment process). In this paper, we do not assume the existence of such rational agent in the market. We show that, in market equilibrium, every agent will survive in the market. More importantly, agents' wealth shares are expected to remain the same under the consensus belief, although they are expected to increase under their own beliefs. Therefore the consensus belief is a fair belief we can use to price option.

Binomial models have been employed to study the pricing of options in the literature. Guidolin and Timmermann (2003) model the dividend growth rate as a binomial lattice with constant probability and rate changes in each state, the former is unknown whereas the latter is known. They also assume there is a representative agent with CRRA utility who updates his belief about the probability of a positive rate change as a Bayesian learner. They find that the stock price under Bayesian learning (BL) with incomplete information does not converge to the Black-Scholes model whereas the convergence occurs under complete information. Call prices under BL with certain priors exhibit implied volatility that resembles the market implied volatility observed in the S\&P500 index options. In another related paper, Guidolin and Timmermann (2007) characterize equilibrium asset prices under adaptive, rational and BL schemes in a model where dividends evolve on a binomial lattice. The properties of equilibrium stock and bond prices under learning are shown to differ significantly.

This paper is also related to literature on incomplete information, model uncertainty and rational learning. Heterogeneous agents with different priors learn rationally from observed quantities (David and Veronesi (2002), Buraschi and Jiltsov (2006) and Li (2007), Cao and Ou-Yang (2009)). David and Veronesi (2002) develop a continuous time model to study option pricing in which the dividend growth rate has two possible states and investors need to determine the current state of the growth rate. Buraschi and Jiltsov (2006) assume that investors observe the dividend process and also a signal that correlates with the growth rate. They use the model to explain open interest in the option market since options are non-redundant in an incomplete market. Li (2007) assumes investors have different time preferences as well as heterogeneous beliefs about the dividend process. Cao and Ou-Yang (2009) analyze the effects of differences of opinion on the dynamics of trading volume in stocks and options. They find that differences in the mean and precision of the terminal stock payoff impact differently on the trading of stocks and options. In general, models with uncertainty and learning provide a better explanation to the observed implied volatilities than models with an exogenous stock price process with respect to economic intuitions and the fitting of the volatility surface.

This paper is organized as follows. Section 2 presents the binomial model with heterogeneous beliefs. Section 3 defines a consensus belief in market equilibrium and shows how the consensus belief can be constructed from investors' subjective beliefs. We also define and identify a fair belief for pricing contingent claims in the market. Section 4 performs a static analysis of the impact of heterogeneous beliefs on the equilibrium price of the risky asset and the risk-free rate in a single-period setting. In section 5, we develop a fair option pricing formula and study the impact of heterogeneous beliefs on the return distribution and the implied volatility of the call prices under the consensus belief numerically. Section 6 concludes.

\section{Model and Heterogeneous Beliefs}

We consider a simple economy with one risky asset and one riskless asset in discrete time, $t=0,1,2, \cdots, T$. 
The risky asset has one share available and does not pay dividends and the riskless asset is in zero net supply for all time $t$. The price $S(t)$ of the risky asset follows a multi-period Cox-Ross-Rubinstein model. More specifically, we assume under agent $i$ 's subjective belief, the return of the risky asset follows

$$
R(t+1)=\frac{S(t+1)}{S(t)}= \begin{cases}u_{i}, & p_{i} \\ d_{i}, & 1-p_{i}\end{cases}
$$

where $u_{i}, d_{i}$ and $p_{i}$ are all constants with the no-arbitrage condition $d_{i}<R_{f}(t)<u_{i}$, where $R_{f}(t)=B(t+$ $1) / B(t)$ is the return of the riskless asset over the period $[t, t+1]$, and $r_{f}(t)=R_{f}(t)-1$ is the risk-free rate. We assume there are $I$ heterogeneous agents in the economy, indexed by $i=1,2, \cdots, I$, where $\mathcal{B}_{i}:=\left(p_{i}, u_{i}, d_{i}\right)$ charaterizes agent $i$ 's belief about the probability distribution and the sizes of the future asset returns.

Let $W_{i}(t)$ be the wealth of agent $i$ and $\omega_{i}(t)$ be the proportion wealth invested in the risky asset at time $t$. Agent $i$ 's objective at time $t=0$ is

$$
\max _{\left\{\omega_{i}(t)\right\}} \mathbb{E}_{0}^{i}\left(U\left(W_{i}(T)\right)\right)
$$

where $U(\cdot)$ is the utility function, $W_{i}(T)$ the portfolio's terminal wealth and $\mathbb{E}_{0}^{i}$ the expectation of agent $i$. For tractability, we assume that all agents have log-utility, $U_{i}(x)=\ln (x)$. Then agent $i$ 's objective in Equation (1) becomes

$$
\max _{\left\{\omega_{i}(t)\right\}}\left\{\ln \left(W_{i}(0)\right)+\sum_{t=0}^{T-1} \mathbb{E}_{0}^{i}\left[\ln \left(R_{f}(t)+\omega_{i}(t)\left(R(t+1)-R_{f}(t)\right)\right)\right]\right\} .
$$

The optimization problem in (2) can be solved using dynamic programming or the martingale approach. ${ }^{4}$ To ease the notations, we have suppressed the time indexes, all model parameters correspond to the time period $[t, t+1]$ unless stated otherwise.

Lemma 2.1. Let $\bar{u}_{i}=u_{i}-R_{f}$ and $\bar{d}_{i}=d_{i}-R_{f}$ be the excess return in the up and down states, respectively over the period $[t, t+1]$ under agent $i$ 's belief. Then the optimal portfolio of agent $i$ 's multi-period optimization problem in (2) is given by

$$
\omega_{i}=R_{f} \frac{\bar{u}_{i} p_{i}+\bar{d}_{i}\left(1-p_{i}\right)}{-\bar{u}_{i} \bar{d}_{i}}
$$

for $t=0,1, \cdots, T-1$.

Lemma 2.1 shows that agent $i$ is able to determine the optimal proportion of his wealth to invest in the risky asset once the risk-free rate at time $t$ is observed. Also the optimal proportion only depends on agent $i$ 's belief about the distribution of asset return in period $[t, t+1]$. Essentially, to maximize the logarithm of a portfolio's terminal wealth is equivalent to maximize the expected growth rate $\mathbb{E}\left[\ln \left(1+R_{p}(t+1)\right)\right]$ period by period, where $R_{p}(t+1)$ is the rate of return of the portfolio from $t$ to $t+1$. This is the so called short-sighted or myopic behavior of logarithmic utility due to the fact that the log-utility maximizers do not consider future investment opportunities in their portfolio selections (see Cvitanic and Zapatero (2004), Chapter 4).

\footnotetext{
${ }^{4}$ Detailed solution to the problem under both methods can be found in Cvitanic and Zapatero (2004) Chapter 4.
} 


\section{Consensus Belief and Market Equilibrium}

In this section, we first introduce a consensus belief and use it to characterize the market equilibrium. We then show that the consensus belief is also a fair belief under which the wealth shares of all the agents are martingale process, although they are sub-martingale under their own beliefs.

With one unit of the risky asset and zero net supply for the riskless asset in the market, in order for the market to clear, agents' total investment in the risky asset must be equal to the aggregate market wealth and hence the price of the risky asset at all times,

$$
\sum_{i=1}^{I} \omega_{i}(t) W_{i}(t)=W_{m}(t)=S(t), \quad t=0,1, \cdots, T-1,
$$

where $W_{m}(t)=\sum_{i=1}^{I} W_{i}(t)$ denotes the aggregate market wealth at time $t$. We refer Equation (4) as the market clearing condition for our economy. Substituting Equation (3) into the market clearing condition (4) leads to the following expression involving the equilibrium risk free rate from time $t$ to $t+1$,

$$
\frac{1}{R_{f}}+\sum_{i} w_{i}\left(\frac{p_{i}}{\bar{d}_{i}}+\frac{1-p_{i}}{\bar{u}_{i}}\right)=0
$$

where $w_{i}=\frac{W_{i}(t)}{W_{m}(t)}$ is the wealth share of agent $i$ at time $t$. Equation (5) shows that the equilibrium risk-free rate depends on the beliefs of all the agents and their wealth shares. Ideally one would like to aggregate agents' heterogeneous beliefs and construct a consensus belief to represent the equilibrium, see Chiarella et al. (2010, 2011) in a static mean-variance setting and Jouini and Napp $(2006,2007)$ in an intertemporal consumption setting. We now introduce a consensus belief for the CRR model with the heterogeneous beliefs.

Definition 3.1. For the CRR model with the heterogeneous beliefs, a belief $\mathcal{B}_{m}:=\left(p_{m}, u_{m}, d_{m}\right)$, defined by the probability of an up move and returns of the risky asset in the up and down states respectively in period $[t, t+1]$ for $t=0,1, \cdots, T-1$, is a consensus belief if the asset price $S$ and the equilibrium risk-free rate $R_{f}$ under the heterogeneous beliefs are the same as those in the economy in which all agents have the homogeneous belief $\mathcal{B}_{m}$.

The introduction of the consensus belief allows the transformation of a market with heterogeneous beliefs to a market under which all agents are identical in their beliefs. Consequently, the market can be characterized by a representative agent with the consensus belief. For the representative agent with log-utility and the consensus belief $\mathcal{B}_{m}$, by the market clearing condition, the risky asset must be the growth optimal portfolio. The following Proposition 3.2 demonstrates how to construct the consensus belief implicitly and shows that the risk-free rate and the consensus belief can be determined simultaneously.

Proposition 3.2. In market equilibrium,

(i) the consensus belief $\mathcal{B}_{m}:=\left(p_{m}, u_{m}, d_{m}\right)$, is given by

$$
p_{m}=\sum_{i} w_{i} p_{i}, \quad u_{m}=\bar{u}_{m}+R_{f}, \quad d_{m}=\bar{d}_{m}+R_{f},
$$

with

$$
\bar{u}_{m}=\left(\sum_{i=1}^{I} w_{i} \frac{1-p_{i}}{1-p_{m}} \bar{u}_{i}^{-1}\right)^{-1}
$$




$$
\bar{d}_{m}=\left(\sum_{i=1}^{I} w_{i} \frac{p_{i}}{p_{m}} \bar{d}_{i}^{-1}\right)^{-1} .
$$

(ii) the equilibrium risk free rate satisfies

$$
\frac{1}{R_{f}}=\frac{1-p_{m}}{d_{m}}+\frac{p_{m}}{u_{m}}=\mathbb{E}_{t}^{m}\left[\frac{1}{R(t+1)}\right]
$$

where $R(t+1)$ is the gross return of the risky asset and $\mathbb{E}_{t}^{m}[\cdot]$ is the conditional expectation of the representative agent under the consensus belief.

(iii) the state prices or the risk neutral probabilities of the up and down states at time $t$ under individual subjective belief $\mathcal{B}_{i}$ and the consensus belief $\mathcal{B}_{m}$ are given by

$$
q_{i, u}(t)=\frac{-\bar{d}_{i}}{\bar{u}_{i}-\bar{d}_{i}}, \quad q_{i, d}(t)=\frac{\bar{u}_{i}}{\bar{u}_{i}-\bar{d}_{i}}, \quad i=1,2, \cdots, I, m .
$$

(iv) the stock price at time $t$ can be written as

$$
S(t)=\frac{\mathbb{E}_{t}^{Q_{i}}(S(t+1))}{R_{f}}=\frac{\mathbb{E}_{t}^{i}\left(\xi_{i} S(t+1)\right)}{R_{f}}, \quad i=1,2, \cdots, I, m,
$$

where

$$
\xi_{i}= \begin{cases}\frac{q_{i, u}(t)}{p_{i}}, & p_{i} ; \\ \frac{q_{i, d}(t)}{1-p_{i}}, & 1-p_{i}\end{cases}
$$

is the Randon-Nikodym derivative that changes the probability measure from $P_{i}$ to $Q_{i}$, which is often referred to as the "pricing kernel" in the asset pricing literatures.

The proof of Proposition 3.2 is given in Appendix A. Proposition 3.2 (i) shows that the consensus belief of the probability of an up move $p_{m}$ is simply an arithmetic average of individual probability beliefs $p_{i}$ weighted by their wealth shares $w_{i}$. This means that the probability $p_{m}$ is more influenced by the wealthier agents. Also the consensus belief of the excess return in each state $\left(\bar{u}_{m}, \bar{d}_{m}\right)$ is a harmonic mean of individual beliefs of the excess returns $\left(\bar{u}_{i}, \bar{d}_{i}\right)$ weighted by their wealth shares and probabilities, hence $\bar{u}_{m}\left(\bar{d}_{m}\right)$ is more influenced by agents who are wealthier and relatively pessimistic (optimistic). More importantly, the consensus belief $\mathcal{B}_{m}$ can only be determined simultaneously by the risk-free rate and the wealth shares of all the agents in the market, $w_{i}(t)$ for $i=1,2, \cdots, I$ and $t=0,1, \cdots, T-1$. Proposition 3.2 (ii) indicates a relationship between the returns of risky and riskless assets under the consensus belief. From Equation (9) it can be deduced that

$$
\mathbb{E}_{t}^{m}\left[\frac{B(t+1)}{S(t+1)}\right]=\frac{B(t)}{S(t)}
$$

i.e., the benchmarked price of the riskless asset is a martingale under the consensus belief. Proposition 3.2 (iii) shows that agents in this economy perceive different state prices, indicated by (10), hence the option prices are different for different agents with different beliefs. However, Proposition 3.2 (iv) indicates that all the agents agree on the current observed asset price though they may have distinctive pricing kernels due to their different beliefs.

One of the important observations from Proposition 3.2 is that all the agents have their own state prices, hence the prices of any contingent claims would be different under each agent's subjective belief $\mathcal{B}_{i}(i=$ 
$1,2, \cdots, I)$ and the consensus belief $\mathcal{B}_{m}$. The question is which belief we should use for pricing contingent claims. ${ }^{5}$ To answer this question, we introduce a "fair" belief to price contingent claims in our economy.

Definition 3.3. Given the information at time $t=0$, a belief $\mathcal{B}^{*}:=\left(p^{*}, u^{*}, d^{*}\right)$ is a fair belief if and only if the wealth share of agent $i$ is a martingale under the belief $\mathcal{B}^{*}$,

$$
\mathbb{E}_{t}^{*}\left[w_{i}(t+1)\right]=w_{i}(t)
$$

for $i=1,2, \cdots, I$ and $t=0,1, \cdots, T-1$.

It follows from the law of iterated expectations that $\mathbb{E}_{t}^{*}\left[w_{i}(T) / w_{i}(t)\right]=1$ under a fair belief. Therefore, the wealth share of every agent is expected to remain at its current level from time $t$ to the terminal time $T$ for all the agents. The belief $\mathcal{B}^{*}$ is fair in the sense that all the agents on average perform equally under this belief when the market is in equilibrium. The next result shows a relationship between the consensus belief and a fair belief.

Proposition 3.4. The consensus belief $\mathcal{B}_{m}$ is a fair belief, that is

$$
\mathbb{E}_{t}^{m}\left[\frac{w_{i}(t+1)}{w_{i}(t)}\right]=1
$$

The proof of Proposition 3.4 is in Appendix B. Proposition 3.4 shows that the consensus belief is a fair belief in the market equilibrium. Intuitively, the consensus belief consists of the beliefs from all the agents and, in equilibrium, the wealth shares of all the agents are expected to be their current wealth shares in the market. However, under their own beliefs, all the agents expect their wealth shares to increase, as demonstrated in the next result.

Proposition 3.5. Under agent i's subjective belief $\mathcal{B}_{i}$,

$$
\mathbb{E}_{t}^{i}\left[\frac{w_{i}(t+1)}{w_{i}(t)}\right] \geqslant 1
$$

for $t=0,1, \cdots, T-1$. The equality holds if and only if

$$
\mathbb{E}_{t}^{i}\left[\frac{1}{R(t+1)}\right]=\mathbb{E}_{t}^{m}\left[\frac{1}{R(t+1)}\right]=\frac{1}{R_{f}} ;
$$

that is when agent $i$ 's belief is the consensus belief.

The proof of Proposition 3.5 is in Appendix C. Using the law of iterated expectation, Proposition 3.5 implies that $\mathbb{E}_{t}^{i}\left[\frac{w_{i}(T)}{w_{i}(t)}\right] \geqslant 1$ for all $t \in[0, T-1]$. This indicates that agent $i$ expects his wealth share to grow, which is what the agent is trying to achieve. This result is not inconsistent with Proposition 3.4 arguing that the expected wealth shares of all the agents are not changed under the fair belief, which is the consensus belief in the market equilibrium. Proposition 3.5 shows that agent $i$ 's subjective belief is fair if and only if the discounted value of a dollar payoff by the stock under his expectation is the same as the zero-coupon bond price. The above analysis demonstrates that, when a contingent claim is priced differently for agents under their own beliefs, the consensus belief $\mathcal{B}_{m}$ is a fair belief to price contingent claims in our economy.

5 Any contingent claims other than the stock or the bond are redundant securities in our economy since the market is complete in the sense that each agent can construct their optimal portfolio by investing in the risk-free asset and the risky asset only. 


\section{Impact of Mean-Preserving Heterogeneous beliefs}

It is often believed that the effect from belief biases such as pessimism and optimism should be cancelled out when the "average" belief is unbiased. In this section, we examine the impact of mean-preserving heterogeneous beliefs on the market consensus belief, the expected return of the risky asset, the equilibrium risk-free rate, and the equity premium. In our setting, with mean-preserving heterogeneous beliefs ${ }^{6}$, the average belief is unbiased. From Proposition 3.2, it is clear that the consensus belief $\mathcal{B}_{m}$ is different from the average belief $\overline{\mathcal{B}}$. We want to see how the expected return of the risky asset, the risk-free rate, and risk premium in the market equilibrium (under the consensus belief $\mathcal{B}_{m}$ ) are different from that under the average belief $\overline{\mathcal{B}}$ for an increasing level of divergence of opinions.

For simplicity, we consider a static setting where agents' wealth shares are equal and their beliefs of the asset return are uniformly distributed for both the up and down states. We only focus on a single time period $[t, t+1]$ where information at time $t$ is known.

Corollary 4.1. Consider a market of I agents with equal initial wealth share $w_{i}(t)=1 / I$ and homogeneous belief in probabilities $p_{i}=p$ for all $i=1,2, \cdots, I$. Let $\left(u_{o}(t), d_{o}(t)\right)$ be a benchmark belief. Assume that the subjective beliefs of agents diverge from the benchmark belief uniformly, $\left(u_{i}(t), d_{i}(t)\right)=\left(u_{o}(t)+\tilde{\epsilon}_{i u}, d_{o}(t)+\tilde{\epsilon}_{i d}\right)$, where $\tilde{\epsilon}_{i u}$ and $\tilde{\epsilon}_{i d}$ are both i.i.d for agent $i$ with mean of zero and a bounded variance. Therefore agents' divergence of opinions regarding the stock returns in both up and down states are i.i.d. It follows from (7) and (8) that the consensus belief is given by

$$
\bar{u}_{m}=\left(\sum_{i=1}^{I}\left(u_{o}-R_{f}+\tilde{\epsilon}_{i, u}\right)^{-1}\right)^{-1} \quad \bar{d}_{m}=\left(\sum_{i=1}^{I}\left(d_{o}-R_{f}+\tilde{\epsilon}_{i, d}\right)^{-1}\right)^{-1} .
$$

In Corollary 4.1, it is clear that the agents have heterogeneous beliefs regarding the future return of the risky asset, while the average belief $\overline{\mathcal{B}}$ approaches the benchmark belief $\mathcal{B}_{o}$ when there is a large number of agents. The question is whether the divergence of opinions has a significant effect on the market consensus belief of the asset return and hence the equilibrium risk-free rate and the equity premium. To answer this question, we conduct a Monte Carlo simulations based on $\left(u_{o}, d_{o}\right)=(1.235,0.905), p=0.5$ and $I=5,000$, meaning that all the agents agree on that the up and down states are equal likely to occur and agent's wealth share $w_{i}=1 / 5000(=0.02 \%)$ for all the agents. Moreover, we assume that $\tilde{\epsilon}_{i, u} \sim \operatorname{Unif}\left(-\theta_{u}, \theta_{u}\right)$ and $\tilde{\epsilon}_{i, d} \sim \operatorname{Unif}\left(-\theta_{d}, \theta_{d}\right)$, in which $\theta_{u}$ and $\theta_{d}$ measure the degree of the divergence in agents' beliefs. Next, we approximate the consensus belief $\mathcal{B}_{m}$ and the risk-free rate $r_{f}$ by Monte-Carlo simulations with various combinations of the parameters $\theta_{u}$ and $\theta_{d}$. Fig. 1 compares the equilibrium expected return of the risky asset, the risk-free rate and the risk premium to the benchmark for different combinations of $\left(\theta_{u}, \theta_{d}\right)$. In the benchmark case, the risk-free rate is $4.5 \%$ and the risk premium equals to $2.5 \%$.

When agents have a homogeneous belief in $d_{i}$ and heterogeneous beliefs in $u_{i}$, Fig. 1 (a1) illustrates that the equilibrium expected stock return decreases as the level of divergence of opinion in the up state $\theta_{u}$ increases. This is because the agents who are pessimistic regarding future stock return in the up state have more impact on the consensus belief in the up state. Note that $u_{m}$ is harmonic mean of the heterogeneous beliefs; it decreases as $\theta_{u}$ increases, though agents' beliefs $u_{i}$ are a mean-preserving spread of the benchmark.

${ }^{6}$ The average belief is defined by $\overline{\mathcal{B}}:=\left(\frac{1}{I} \sum_{i=1}^{I} p_{i}, \frac{1}{I} \sum_{i=1}^{I} u_{i}, \frac{1}{I} \sum_{i=1}^{I} d_{i}\right)$. 

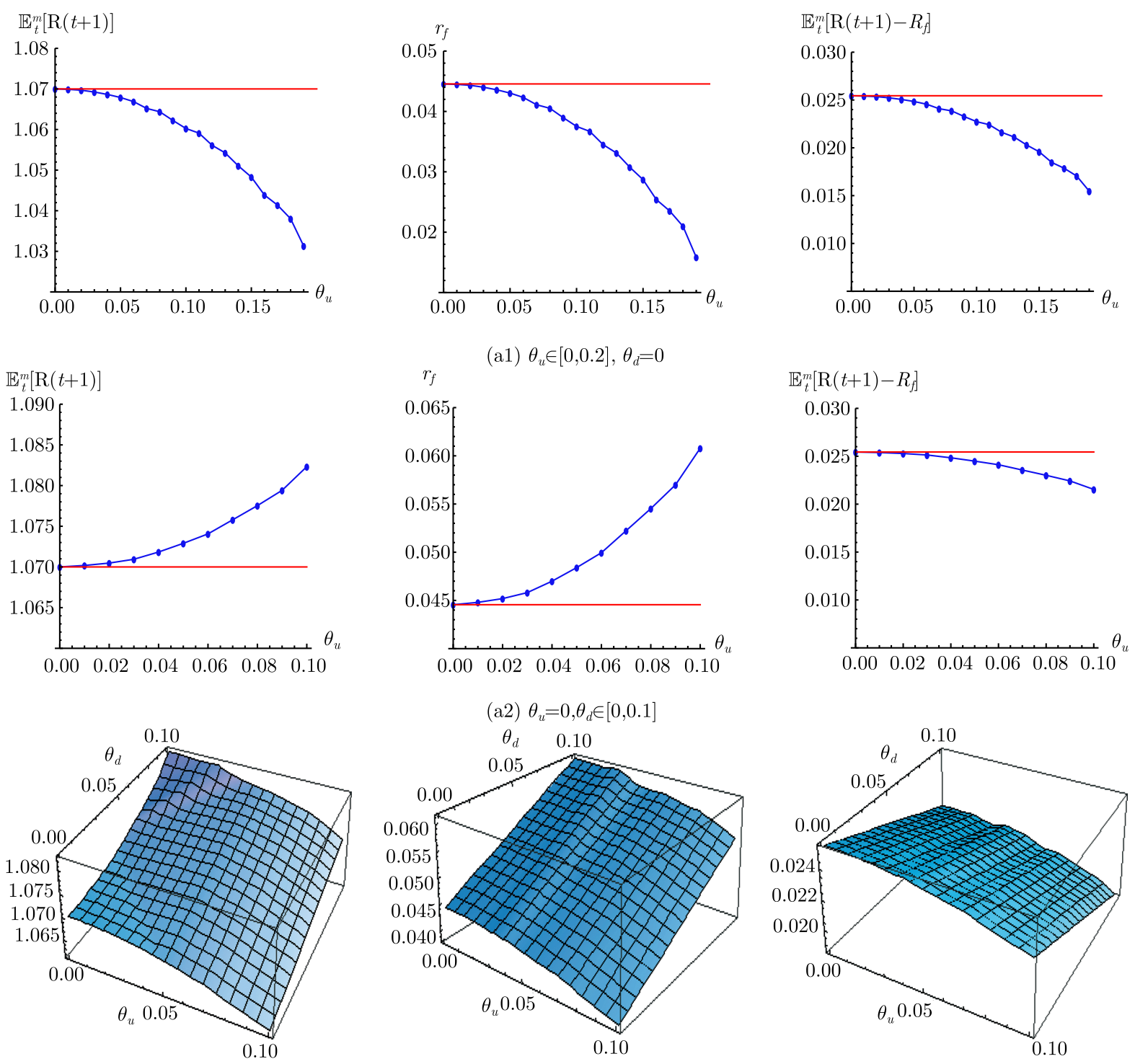

(a3) $\theta_{u}=\in[0,0.1], \theta_{d} \in[0,0.1]$

Fig. 1 Impact of Divergence of Opinion on the Equilibrium Consensus Belief $\mathcal{B}_{m}=\left(u_{m}, d_{m}\right)$, Expected Stock Return $\mathbb{E}_{t}^{m}[R(t+1)]=p u_{m}+(1-p) d_{m}$, the Risk-Free Rate $r_{f}=R_{f}-1$, and the Risk Premium $\mathbb{E}_{t}^{m}\left[R(t+1)-R_{f}\right]$

Therefore the aggregate market is less willing to invest in the risky asset and more willing to invest in the risk-free rate, which reduces the expected return of the risky asset and the risk-free asset compared to the benchmark. The risk premium $\mathbb{E}\left[R(t+1)-R_{f}\right]$ is negatively related to the divergence of opinions, suggesting that, under the consensus belief, the reduction in the risk-free rate is less than that in the expected stock return. When the agents have a homogeneous belief in $u_{i}$ and heterogeneous beliefs in $d_{i}$, Fig. 1 (a2) shows that the expected stock return increases as the level of divergence of opinion $\theta_{d}$ increases. Since the optimistic agents who perceive higher stock return in the down state have more impact on the consensus belief in the down state, $d_{m}$ increases though agents' beliefs $d_{i}$ are mean-preserving spread of the benchmark. In this case the aggregate 
market is more willing to invest in the risky asset and less willing to invest in the risk-free asset, which increases the risk-free rate from the benchmark. Similarly, the risk premium $\mathbb{E}_{t}^{m}\left[R(t+1)-R_{f}\right]$ is negatively related to divergence of opinions, suggesting that the increase in the expected stock return is not enough compare to the increase in the risk-free rate. When we combine the divergence of the opinion in the up and down states, Fig. 1 (a3) shows the combined effect of $\theta_{u}$ and $\theta_{d}$ on the expected stock return, risk-free rate and the risk premium. It is clear that $\theta_{u}$ and $\theta_{d}$ have opposite effect on the expected stock return and the risk-free rate, however they both have a negative effect on the risk premium.

\section{Option Pricing under Heterogeneous Beliefs}

In this section, we examine how the heterogeneous beliefs can be used to explain volatility skewness observed in option markets. As discussed in the previous section, when agents have different beliefs, they have different state prices and therefore they price options differently. In Section 3, we have introduced a fair belief and shown that the consensus belief is a fair belief. We also argue that the option should be priced under the consensus belief, which is the approach used when we price option in this section. We first present a (fair) option pricing formula under the consensus belief $\mathcal{B}_{m}$ and then examine the impact of heterogeneous beliefs on the option prices and implied volatility.

Proposition 5.1. Given the information at time $t$, the fair price of an option $V(t, S(t))$ with payoff function $H(T, S(T))$ is given by

$$
V(t, S(t))=S(t) \mathbb{E}_{t}^{m}(H(T, S(T)) / S(T))
$$

The proof of Proposition 5.1 is in Appendix D. To examine the impact of the heterogeneous beliefs on the option value, we consider the following numerical example. We use the pricing formula developed in Proposition 5.1 to price European call options with different strikes written on the risky asset.

Example 5.2. Assume there are two agents $(i=1,2)$ whose beliefs $\mathcal{B}_{i}$ are characterized by

$$
\begin{aligned}
u_{i}(t) & =1+\mu_{i} \Delta+\sigma_{i} \sqrt{\Delta}, \\
d_{i}(t) & =1+\mu_{i} \Delta-\sigma_{i} \sqrt{\Delta}, \\
p_{i}(t) & =0.5 \\
w_{i}(0) & =0.5
\end{aligned}
$$

where $t=0,1, \cdots, T-1, \Delta=T / n$ and $n$ is the number of trading periods from time 0 to $T$.

In Example 5.2, both agents agree that the stock price is equally likely to move up or down and the relative price changes are constant over each time period from time 0 to $T$. However, they disagree on the sizes of the relative price changes, which are characterized by parameters $\mu_{i}$ and $\sigma_{i}$, agent $i$ 's belief about the expected return and volatility of the stock. Both $\mu_{i}$ and $\sigma_{i}$ are per annum, compounded $n$ periods a year. Obviously, as the number of the trading period $n$ approaches to infinity, the stock price under agent $i$ 's belief converges weakly to the stochastic differential equation ${ }^{7}$

$$
d S(t) / S(t)=\mu_{i} d t+\sigma_{i} d Z_{i}(t)
$$

7 See Kloeden and Platen (1992) and Nelson and Ramaswamy (1990) 
where $Z_{i}(t)$ is a Wiener process under agent $i$ 's belief.

Remark 5.3. In Example 5.2, when $\mu_{i}=\mu$ and $\sigma_{i}=\sigma$ for $i=1,2$ and $n$ approaches to infinity, the price of a call option on the risky asset is given by the Black-Scholes formula and the instantaneous risk-free rate is constant and given by $\mu-\sigma^{2}$.

The proof of Remark 5.3 is in Appendix E. This remark suggests that as the number of trading period $n \rightarrow \infty$, the risk-free rate becomes a constant, depending on the expected return $\mu$ and volatility $\sigma$ of the stock. Furthermore, the call option prices are given by the Black-Scholes formula using $r_{f}=\mu-\sigma^{2}$.

We now consider the case where two agents differ in their beliefs of the growth rate and the volatility of the stock. More specifically, we assume that $\left(\mu_{1}, \mu_{2}\right)=\left(\mu_{o}+\delta_{\mu}, \mu_{o}-\delta_{\mu}\right)$ and $\left(\sigma_{1}, \sigma_{2}\right)=\left(\sigma_{o}+\delta_{\sigma}, \sigma_{o}-\delta_{\sigma}\right)$ such that $\mu_{o}$ and $\sigma_{o}$ are the arithmetic average beliefs of agents' expected return and volatility of the future stock returns respectively. Note that $\delta_{\mu}>0$ indicates that agent 1 is relatively more optimistic than agent 2, believing in a higher expected return, while $\delta_{\sigma}>0$ indicates that agent 1 is less confident than agent 2 , believing a higher volatility. We obtain distribution of the log stock price under the consensus belief at time $T$ and compute the European call option prices via Monte Carlo simulations using Proposition 5.1. The benchmark belief is given by $\left(\mu_{o}, \sigma_{o}\right)=(0.07,0.225)$. Time to maturity $T=0.25$ and time increment $\Delta=0.00025$. Under the consensus belief $\mathcal{B}_{m}$, the binomial tree for the future stock prices is non-recombining ${ }^{8}$ though it is recombining under each agent's belief. We therefore use Monte Carlo simulations for evaluation of the option prices $C_{m}(K)$. Furthermore, it is computationally very expensive to generate stock price path since for each path, we need to numerically solve for the risk-free rate at each time step. Therefore, we use the Black-Scholes option price as a control variate to reduce the variance of the simulated option payoffs, given that the option payoffs under the Black-Scholes model at time $T$ are strongly correlated with the ones under the consensus belief, with correlation is estimated to be close to 1 . Using this technique, we are able to reduce the standard deviation associated with the option payoffs by up to ten times. After obtaining the call option prices under the consensus belief, we then calculate the implied volatilities $\sigma_{i m p}$ using the Black-Scholes option price such that

$$
C_{m}(K)=B S\left(S(0), \sigma_{i m p}, r_{f}, T\right)
$$

where $K$ is the strike price, $S(0)$ is the current stock price, $T$ is the time to maturity, $r_{f}$ is the current risk-free rate, and $C_{m}(K)$ is the fair price of the call option with strike $K$.

With the heterogeneous beliefs, we report the sample statistics of the $\log$ stock price at maturity $\left(\ln \left(S_{T}\right)\right)$ in Table 1 and the distribution of the $\log$ stock price at maturity $\left(\ln \left(S_{T}\right)\right)$ in Fig. 2. In Fig. 2, we also show the distribution of the $\log$ stock price at maturity $\left(\ln \left(S_{T}\right)\right)$ under agent 1 and 2's subjective beliefs $\left(\mathcal{B}_{1}\right.$ and $\left.\mathcal{B}_{2}\right)$, compared to the histogram distribution under the consensus belief $\left(\mathcal{B}_{m}\right)$. Using the Black Scholes option pricing formula, we then calculate and report the implied volatilities for different strike prices in Fig. 3. By normalizing the current stock price to $1, \ln \left(S_{T}\right)$ then measures the continuous return in the period $[0, T]$, which is normally distributed with mean $\left(\mu_{i}-\frac{1}{2} \sigma_{i}^{2}\right) T$ and standard deviation $\sigma_{i} \sqrt{T}$ under the subjective belief of agent $i$. If we interpret the expected log price as the growth rate of the stock, then the agents agree on the expected stock return but perceive different growth rates.

\footnotetext{
8 Non-recombining means that an up move follow by a down move is not the same as a down move follow by an up move. For a non-recombining tree, there are $2^{1000}$ possible values for the stock price after 1000 steps.
} 
Table 1. Impact of the Heterogeneous Beliefs in the Growth Rate $(\mu)$ and the Volatility $(\sigma)$ of Future Stock Returns on the Distribution of the Log Stock Price at Maturity $\left(\ln \left(S_{T}\right)\right)$ for $T=0.25$. The First and Second Columns in Each Table Provide the First 4 Moments of the Distribution of $\ln \left(S_{T}\right)$ under the Subjective Beliefs of Agent 1 and 2 Respectively and the Third Column of Each Table Correspond to That under the Consensus Belief $\mathcal{B}_{m}$.

\begin{tabular}{cccc}
\hline & $\mathcal{B}_{1}$ & $\mathcal{B}_{2}$ & $\mathcal{B}_{m}$ \\
\hline mean & 0.024 & -0.0001 & 0.016 \\
std & 0.1125 & 0.1125 & 0.1099 \\
skew & 0 & 0 & 0.003 \\
kurt & 3 & 3 & 2.874 \\
\hline \multicolumn{4}{c}{$(\mathrm{c} 1)\left(\delta_{\mu}, \delta_{\sigma}\right)=(0.05,0)$} \\
\hline mean & 0.027 & $\mathcal{B}_{2}$ & $\mathcal{B}_{m}$ \\
std & 0.075 & -0.00625 & 0.019 \\
skew & 0 & 0.15 & 0.098 \\
kurt & 3 & 0 & -0.159 \\
\hline
\end{tabular}

(c3) $\left(\delta_{\mu}, \delta_{\sigma}\right)=(0.05,-0.075)$

\begin{tabular}{cccc}
\hline & $\mathcal{B}_{1}$ & $\mathcal{B}_{2}$ & $\mathcal{B}_{m}$ \\
\hline mean & 0.006 & 0.015 & 0.017 \\
std & 0.15 & 0.075 & 0.095 \\
skew & 0 & 0 & -0.138 \\
kurt & 3 & 3 & 3.326 \\
\hline \multicolumn{4}{c}{$(\mathrm{c} 2)\left(\delta_{\mu}, \delta_{\sigma}\right)=(0,0.075)$} \\
\hline mean & $\mathcal{B}_{1}$ & $\mathcal{B}_{2}$ & $\mathcal{B}_{m}$ \\
std & 0.01875 & 0.002 & 0.003 \\
skew & 0.15 & 0.075 & 0.105 \\
kurt & 0 & 0 & 0.100 \\
\hline
\end{tabular}
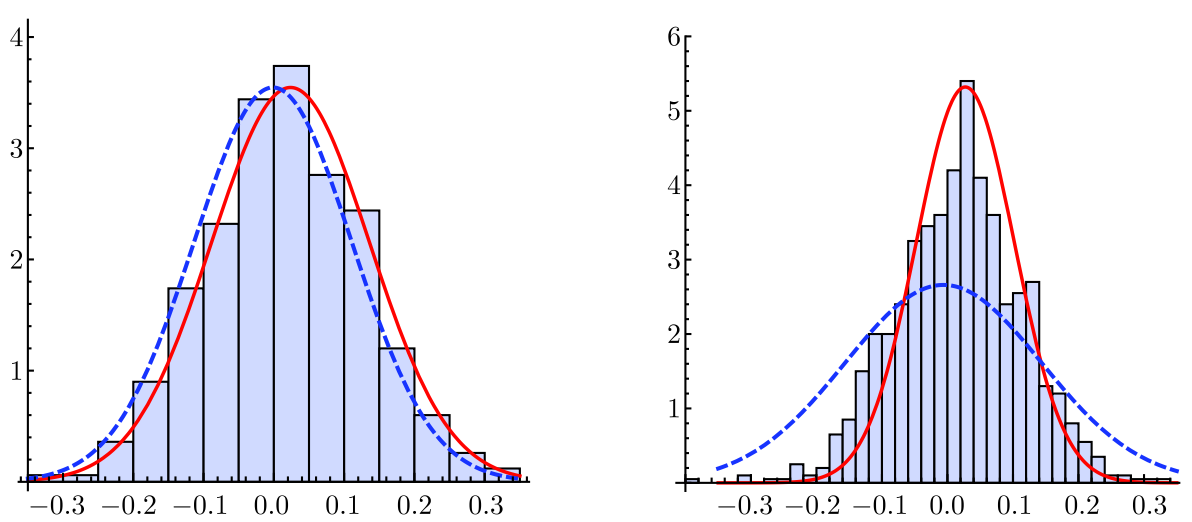

(b1) $\left(\delta_{\mu}, \delta_{\sigma}\right)=(0.50,0)$
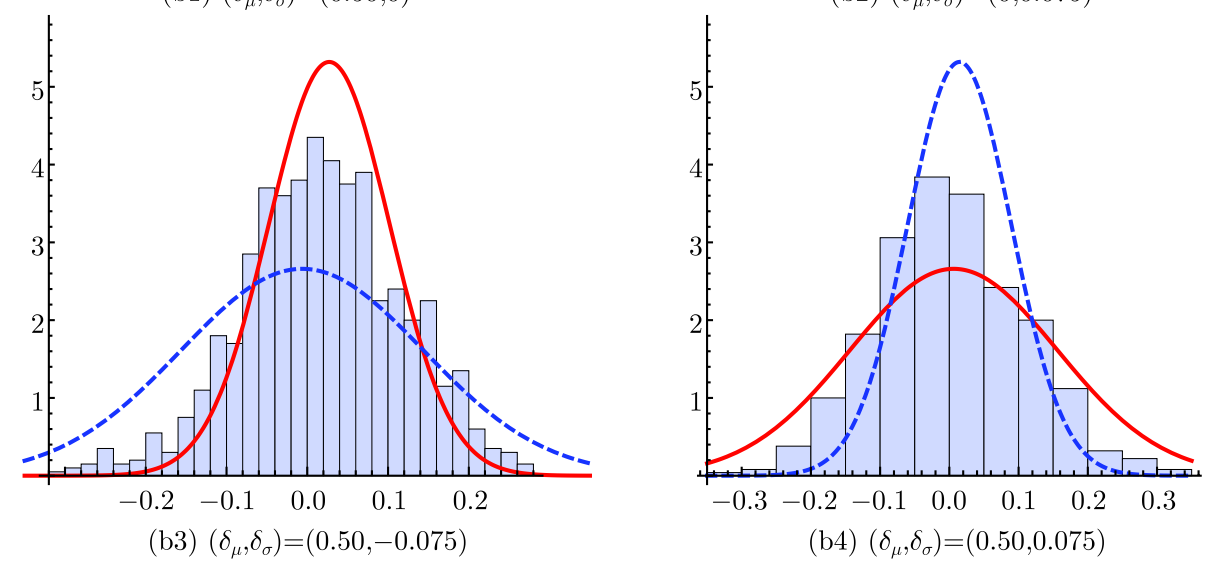

Fig. 2 Impact of the Heterogeneous Beliefs in the Growth Rate $(\mu)$ and the Volatility $(\sigma)$ of Future Stock Returns on the Distribution of the Log Stock Price at Maturity $\left(\ln \left(S_{T}\right)\right)$ for $T=0.25$. The Solid and Dashed Lines Represent the Perceived Distribution of $\ln \left(S_{T}\right)$ by Agent 1 and 2 Respectively and Histogram Represents the Distribution of $\ln \left(S_{T}\right)$ under the Consensus Belief $\mathcal{B}_{m}$ 

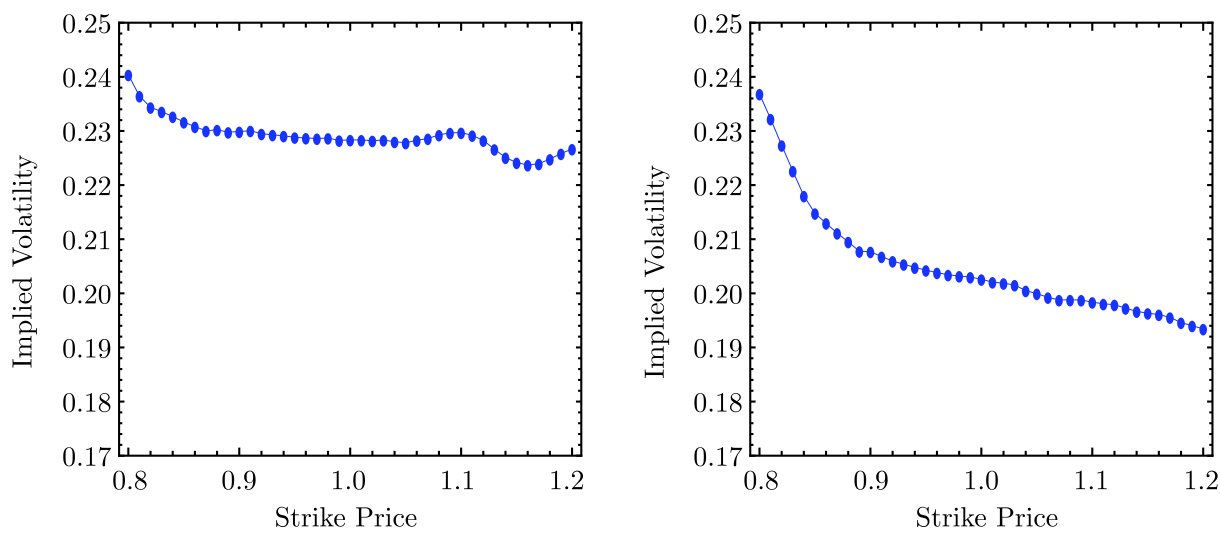

(d1) $\left(\delta_{\mu}, \delta_{\sigma}\right)=(0.50,0)$

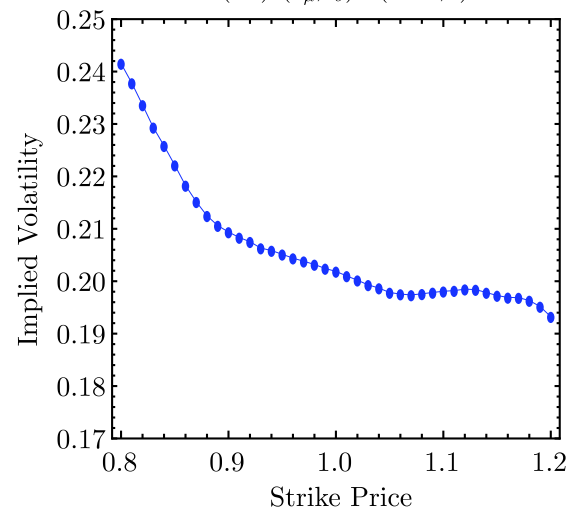

(d2) $\left(\delta_{\mu}, \delta_{\sigma}\right)=(0,0.075)$

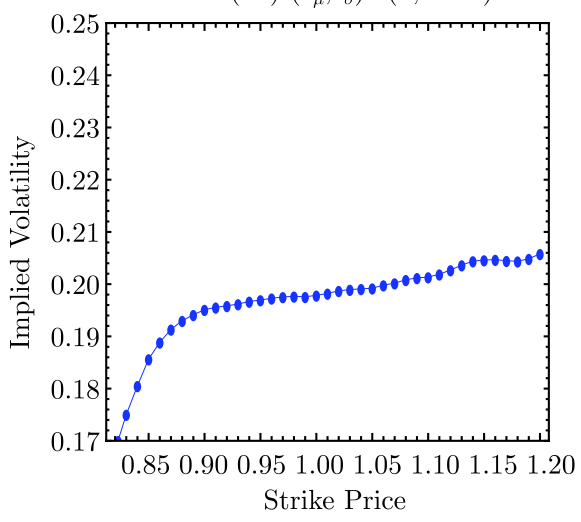

(d3) $\left(\delta_{\mu}, \delta_{\sigma}\right)=(0.50,-0.075)$

(d4) $\left(\delta_{\mu}, \delta_{\sigma}\right)=(0.50,0.075)$

Fig. 3 Impact of the Heterogeneous Beliefs in the Growth Rate $(\mu)$ and the Volatility $(\sigma)$ of Future Stock Returns on the Implied Volatilities of Fair Call Prices

We first examine the impact of the heterogeneous beliefs in the expected return of the stock while agents have a homogeneous belief on the stock return volatility. That is the agents agree on the volatility but disagree on the expected stock return. The return distribution in Fig. 2 (b1) and the statistics in Table 1 (c1) demonstrate that the growth rate under the consensus belief $\mathcal{B}_{m}$ is between that of both agents but closer to that of agent 1 's (the more optimistic agent). However the volatility is close to the common belief. Moreover the distribution of $\ln \left(S_{T}\right)$ is approximately normal under $\mathcal{B}_{m}$ since the skewness is close to zero and the kurtosis is close to 3 . In this case, Fig. 3 (d1) shows that the implied volatility is almost flat with respect to the strike, indicating that the call prices are consistent with the BS formula. This analysis indicates that the heterogeneous beliefs in the expected return only seems not able to explain the volatility skewness observed in option markets. ${ }^{9}$

Fig. 2 (b2) and Table 1 (c2) show that when agents agree on the expected return but disagree on the volatility, $\ln \left(S_{T}\right)$ becomes negatively skewed under the consensus belief $\mathcal{B}_{m}$, the growth rate and volatility under $\mathcal{B}_{m}$ are closer to agent 2's belief, who is more confident. Furthermore, the market perceives a higher growth rate than both agents. Fig. 3 (d2) shows that the implied volatility exhibits a positive skewness, which is consistent with the observed pattern in option markets. Intuitively, since agent 2 perceives a higher growth

${ }^{9}$ Note that this result is partially due to the choice of the log-utility in this paper. With other utility function and time preference, it is possible to explain the volatility skewness even when agents agree to disagree on the expected return only. 
rate, the agent has a larger wealth share and dominates the consensus belief in the upper part of the binomial lattice which matters more for pricing out of the money (OTM) call options (calls with strikes above the current spot price). This means that the OTM call prices would reflect more of agent 2's belief about the stock volatility. Also, since agent 2 perceives a lower stock volatility, the OTM call prices have lower implied volatilities than at the money (ATM) and in the money (ITM) call options. As one moves gradually towards the lower part of the tree, agent 1's belief becomes more and more important in determining the consensus belief. Therefore the implied volatility also increases as the strike prices decreases.

Fig. 2 (b3) and Table 1 (c3) show that when agent 1 is more optimistic and confident than agent 2 , the consensus belief of the growth rate and volatility of the stock are closer to those under agent 1's belief. The distribution of $\ln \left(S_{t}\right)$ is negatively skewed. Fig. 3 (d3) indicates that the call prices exhibit volatility skewness. The intuition is similar to the previous case.

When agent 1 is more optimistic but less confident about future stock returns than agent 2, Fig. 2 (b4) and Table 1 (c4) show that the growth rate and volatility under the consensus belief are closer to those under agent 2's belief who is pessimistic and more confident. The distribution of $\ln \left(S_{T}\right)$ is positively skewed. Fig. 3 (d4) shows that the OTM call prices have higher implied volatilities than the ATM and ITM call options. Intuitively, agent 1 is more optimistic and dominates the consensus belief in the upper part of the tree as previously discussed. However agent 1 perceives a larger volatility than agent 2. Therefore the implied volatilities are positively related to the strike prices.

In order to examine the term structure of the implied volatilities, we calculate the implied volatilities from the fair prices of the call options with time to maturity $T \in[0.25,1.00]$ for $\delta_{\mu}=0.05$ and $\delta_{\sigma}=-0.075$. Fig. 4 shows that the implied volatility surface flattens out as the time to maturity increases. This is consistent with the observed patterns in option market data. This indicates that the fair option prices can mimic the important features of option market data, though Example 5.2 is a very simple specification of our model. We expect the volatility surface generated from the fair option prices under the consensus belief to exhibit even richer patterns with more general specifications.

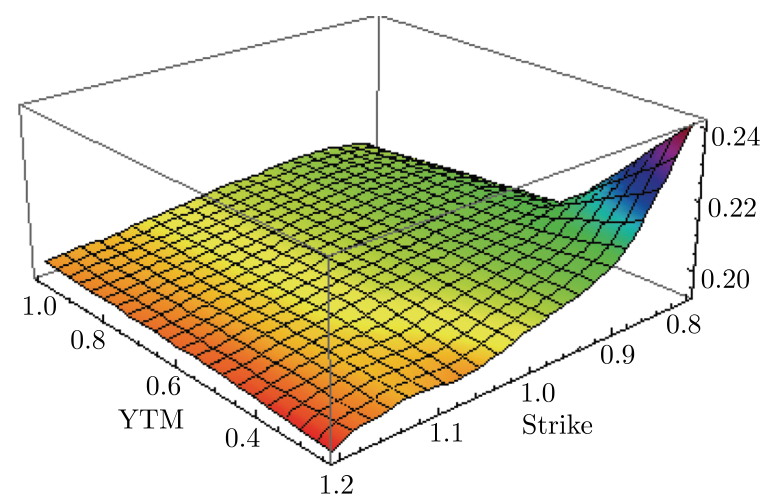

Fig. 4 The Implied Volatilities Calculated with Fair Prices of a Call Option with Time to Maturity $T \in[0.25,1.00]$. The Current Stock Price is $S(t)=1$, the Difference in Agents' Belief is Characterized by $\delta_{\mu}=0.05$ and $\delta_{\sigma}=-0.075$

Example 5.2 can be generalized to take into account other popular stochastic processes for modelling stock 
prices dynamics. In general, if the belief of agent $i$ is characterized by

$$
\begin{aligned}
& u_{i}(t)=1+\mu_{i}(t, S) \Delta+\sigma_{i}(t, S) \sqrt{\Delta} \\
& d_{i}(t)=1+\mu_{i}(t, S) \Delta-\sigma_{i}(t, S) \sqrt{\Delta} \\
& p_{i}(t)=0.5
\end{aligned}
$$

where $t=0,1, \cdots, T-1$ and $\Delta=T / n$ is the time increment. Then as $n$ approaches to infinity, the above characterization implies that agent $i$ believes that the stock price dynamics is describe by

$$
d S(t) / S(t)=\mu_{i}(t, S(t)) d t+\sigma_{i}(t, S(t)) d W(t)
$$

Therefore, in principle our option pricing formula can take into account not only disagreement in model parameters, but also differences in the model structure.

\section{Conclusions}

In this paper, we use the equilibrium asset pricing approach and provide an aggregation method of heterogeneous beliefs within a multi-period binomial lattice framework in market equilibrium. The heterogeneity is characterized by the differences in agents' beliefs about the probability of an up move in each period and also the relative size of the price changes in each period. Agents are bounded rational in the sense that they invest in the growth optimal portfolio based on their own subjective belief. To analyze the impact of the heterogeneity, we introduce the concept of a consensus belief, which relates the heterogeneous market to an equivalent homogeneous market under the consensus belief. The consensus belief is basically a wealth weighted average of agents' subjective beliefs and can be determined simultaneously with the risk-free rate. Through various numerical examples, we have examined the impact of the heterogenous beliefs on the equilibrium risk-free rate, equity risk premium and option prices. In a static analysis when the agents have the equal wealth shares and agree on the probability, the market expects a lower (higher) expected return when the disagreement regarding the future stock return in the up (down) state is high. Also the risk-free is negatively (positively) related to the disagreement about the future return in the up (down) state, while the risk premium is negatively related to the disagreement in general. We also find that the consensus belief is a fair belief in the sense that the expected wealth shares of all the agents are not changed under the consensus belief, but their wealth shares are expected to increase under their own beliefs. When options are priced under the consensus belief, the implied volatilities exhibit volatility skew observed in option markets, in particular when agents disagree only on the volatility of the future stock returns or when the optimistic agent is also confident about the future stock returns.

The binomial model under heterogeneous beliefs developed in this paper can take into account of agents' disagreements in both model parameters and model structure. Future avenues of research include incorporating disagreements in model structures into option pricing and compare the fair option prices implied by agents' beliefs ${ }^{10}$ and those option prices observed in financial market to quantify the level of mispricing. It would be also interesting to extend the current model to include multiple assets including the bond and currency markets.

Acknowledgments: Financial support from the Australian Research Council (ARC) under Discovery Grant (DP130103210) and the National Natural Science Foundation of China (NSFC) Grant (71320107003) is gratefully acknowledged. The usual caveats apply.

Conflicts of Interest: The authors declare no conflict of interest.

10 Analyst forecasts can act as a proxy for agents' belief about future asset returns, a stock index can be taken as the risky asset. 


\section{Appendix A. Proof of Proposition 3.2}

We start from Equation (5)

$$
\frac{1}{R_{f}}+\sum_{i} w_{i}(t)\left(\frac{p_{i}}{\bar{d}_{i}}+\frac{1-p_{i}}{\bar{u}_{i}}\right)=0 .
$$

On the one hand, if every investor has identical belief about the future return in up and down states respectively in period $[t, t+1]$, i.e $p_{i}=p_{m}, \bar{u}_{i}=\bar{u}_{m}$ and $\bar{d}_{i}=\bar{d}_{m}$ for all $i$. Then it is obvious that Equation (5) becomes

$$
\frac{1}{R_{f}}+\frac{p_{m}}{\bar{d}_{m}}+\frac{1-p_{m}}{\bar{u}_{m}}=0
$$

Solving Equation (A1) for $R_{f}$ leads to the relationship between the consensus belief and the the risk-free rate in (9). Next it is obvious that in order for $\mathcal{B}_{m}$ to be the consensus belief, the following must hold in every period $[t, t+1]$ for $t=0,1, \cdots, T$,

$$
\begin{aligned}
\frac{p_{m}}{\bar{d}_{m}} & =\sum_{i} w_{i} \frac{p_{i}}{\bar{d}_{i}}, \\
\frac{1-p_{m}}{\bar{u}_{m}} & =\sum_{i} w_{i} \frac{1-p_{i}}{\bar{u}_{i}},
\end{aligned}
$$

which lead to Equations (7) and (8). When investors agree on the future return in each state, then the consensus belief must reflect this common belief. This means that $u_{i}=u_{o} \Rightarrow u_{m}=u_{o}$ and $d_{i}=d_{o} \Rightarrow d_{m}=d_{o}$. This fact gives us the expression for the consensus probability belief $p_{m}=\sum_{i} w_{i} p_{i}$.

To prove (iv), we simply substitute Equation (12) into the right hand side of Equation (11), then we find that under the belief of agent $i$,

$$
\left[S(t) / R_{f}(t)\right]\left(q_{i, u}(t) u_{i}+q_{i, d}(t) d_{i}\right)=S(t) .
$$

Since $q_{i, u}(t) u_{i}+q_{i, d} d_{i}(t)=R_{f}(t)$, we find that the relation holds for all agent $i$, hence it must also hold for the representative agent.

\section{Appendix B. Proof of Proposition 3.4}

The wealth for agent $i$ at time $t+1$ is given by

$$
W_{i}(t+1)=W_{i}(t)\left(\omega_{i}(t) R(t+1)+\left(1-\omega_{i}(t)\right) R_{f}\right) .
$$

Dividing the aggregate market wealth $W_{m}(t+1)$ on both sides and using the fact that $W_{m}(t+1)=W_{m}(t) R(t+1)$,

$$
w_{i}(t+1)=w_{i}(t)\left(\omega_{i}(t)+\left(1-\omega_{i}(t)\right) \frac{R_{f}}{R(t+1)}\right) .
$$

Taking the expectation under the consensus belief on both sides leads to

$$
\mathbb{E}_{t}^{m}\left[\frac{w_{i}(t+1)}{w_{i}(t)}\right]=\omega_{i}(t)+\left(1-\omega_{i}(t)\right) \mathbb{E}_{t}^{m}\left[\frac{R_{f}}{R(t+1)}\right]
$$

By Proposition 3.2 (ii), $\mathbb{E}_{t}^{m}\left[R_{f} / R(t+1)\right]=1$ and this completes the proof. 


\section{Appendix C.Proof of Proposition 3.5}

Similar to the proof of Proposition 3.4, we have Equation (B1), however, the expectation is taken under agent $i$ 's belief,

$$
\mathbb{E}_{t}^{i}\left[\frac{w_{i}(t+1)}{w_{i}(t)}\right]=\omega_{i}(t)+\left(1-\omega_{i}(t)\right) \mathbb{E}_{t}^{i}\left[\frac{R_{f}}{R(t+1)}\right]
$$

Using Lemma 2.1 to expand the above expression leads to

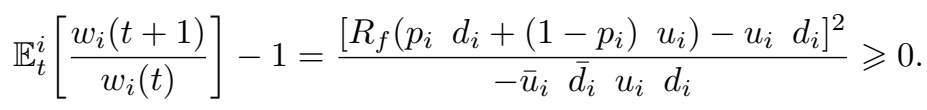

The equality holds if and only if the numerator is zero, that is

$$
\begin{aligned}
& R_{f}\left(p_{i} d_{i}+\left(1-p_{i}\right) u_{i}\right)=u_{i} d_{i} \\
\Rightarrow & \frac{p_{i}}{u_{i}}+\frac{\left(1-p_{i}\right)}{d_{i}}=\frac{1}{R_{f}} .
\end{aligned}
$$

Hence we must have $\mathbb{E}_{t}^{i}\left[\frac{1}{R(t+1)}\right]=\mathbb{E}_{t}^{m}\left[\frac{1}{R(t+1)}\right]=\frac{1}{R_{f}}$.

\section{Appendix D. Proof or Proposition 5.1}

Since the market is complete, we can always replicate the option with a portfolio that invests $\omega(t)$ in the risky asset and $1-\omega(t)$ in the risk-free asset. This means that we can express the value of the option at time $t+1$ as

$$
V(t+1, S(t+1))=V(t, S(t))\left(R_{f}(t)+\omega(t)\left(R(t+1)-R_{f}(t)\right)\right) .
$$

Dividing by $S(t+1)$ on both sides and taking the expectation under the consensus belief yield

$$
\begin{aligned}
\mathbb{E}_{t}^{m}\left(\frac{V(t+1, S(t+1))}{S(t+1)}\right) & =\frac{V(t, S(t))}{S(t)} \mathbb{E}_{t}^{m}\left(\omega(t)+(1-\omega(t)) \frac{R_{f}(t)}{R(t+1)}\right) \\
& =\frac{V(t, S(t))}{S(t)} .
\end{aligned}
$$

Then using the law of iterated expectations,

$$
\frac{V(t, S(t))}{S(t)}=\mathbb{E}_{t}^{m}\left(\frac{V(t+1, S(t+1))}{S(t+1)}\right)=\mathbb{E}_{t}^{m}\left(\frac{V(T, S(T))}{S(T)}\right) .
$$

Note that $V(T, S(T))=H(T, S(T)$. This completes the proof.

\section{Appendix E. Proof of Remark 5.3}

Since both agents have identical beliefs about the distribution of future asset returns, the consensus belief must coincide with the homogeneous belief, that is

$$
\begin{aligned}
& u_{m}(t)=1+\mu \Delta+\sigma \sqrt{\Delta}, \\
& d_{m}(t)=1+\mu \Delta-\sigma \sqrt{\Delta}, \\
& p_{m}(t)=0.5
\end{aligned}
$$

for $t=0,1, \cdots, T-1$. By Proposition 3.2 (ii), the price of a zero-coupon bond is given by

$$
\frac{1}{R_{f}}=\frac{1}{2}\left[\frac{1}{1+\mu \Delta+\sigma \sqrt{\Delta}}+\frac{1}{1+\mu \Delta-\sigma \sqrt{\Delta}}\right]=\frac{1+\mu \Delta}{(1+\mu \Delta)^{2}-\sigma^{2} \Delta} .
$$


Let $r$ be the continuous compounded risk-free rate, we have from the above that

$$
R_{f}=e^{r \Delta}=1+\frac{\mu \Delta-\sigma^{2} \Delta}{1+\mu \Delta} .
$$

Therefore the instantaneous risk-free rate $r_{f}$ is given by

$$
r_{f}=\lim _{\Delta \rightarrow 0} \frac{1}{\Delta} \ln \left[1+\mu \Delta-\frac{\sigma^{2} \Delta}{1+\mu \Delta}\right] \text {. }
$$

By applying $L^{\prime}$ Hôpital's rule we obtain,

$$
r_{f}=\mu-\lim _{\Delta \rightarrow 0} \frac{\sigma^{2}}{(1+\mu \Delta)^{2}\left(1+\mu \Delta-\frac{\sigma^{2} \Delta}{1+\mu \Delta}\right)}=\mu-\sigma^{2}
$$

\section{References}

Abel, A., 2002. An exploration of the effects of pessimism and doubt on asset returns. Journal of Economic Dynamics and Control 26, 1075-1092.

Basak, S., 2000. A model of dynamic equilibrium asset pricing with heterogeneous beliefs and extraneous beliefs. Journal of Economic Dynamics and Control 24, 63-95.

Basak, S., 2005. Asset pricing with heterogeneous beliefs. Journal of Banking and Finance 29, 2849-2881.

Bates, D., 1991. The crash of '87: Was it expected? The evidence form option markets. Journal of Finance 46, $1009-1044$.

Berraday, T., 2009. Bounded rationality and asset pricing with intermediate consumption. Review of Finance 13, 693725 .

Blume, L., Easley, D., 2006. If you are so smart, why aren't you rich? Belief selection in complete and incomplete markets, Econometrica 74, 929-966.

Buraschi, A., Jiltsov, A., 2006. Model uncertianty and option markets with heterogeneous beliefs. Journal of Finance $61,2814-2897$.

Cao, H., Ou-Yang, H., 2009. Differences of opinion of public information and speculative trading in stocks and options. Review of Financial Studies 22, 299-335.

Chiarella, C., Dieci, R., He, X., 2010. A framework for CAPM with heterogeneous beliefs, in: Bishi, G.I., Chiarella, C., Gardini, L. (Eds), Nonlinear Dyanmaics in Economics, Finance and Social Sciences: Essays in Honour of John Barkley Rosser Jr. Springer-Verlag Berlin Heidelberg, Germany, pp. 353-369.

Chiarella, C., Dieci, R., He, X., 2011. Do heterogeneous beliefs diversity market risk? European Journal of Finance $17,241-258$.

Cox, J., Ross, S., Rubinstein, M., 1979. Option pricing: A simplified apporach. Journal of Financial Economics 7, 229263.

Cvitanic, J., Zapatero, F., 2004. Introduction to the Economics and Mathematics of Financial Markets. The MIT Press, Cambridge, MA, USA.

David, A., 2008. Heterogeneous beliefs, speculation, and the equity premium. Journal of Finance 63, 41-83.

David, A., Veronesi, P., 2002. Options under Uncertain Fundamentals. Working Paper, Univeristy of Chicago, Chicago, IL, USA.

DeLong, J., Shleifer, A., Summers, L., Waldmann, R., 1990. Noise trader risk in financial markets. Journal of Political Economy 98, 703-738. 
DeLong, J., Shleifer, A., Summers, L. and Waldmann, R., 1991. The survival of noise traders in financial markets. Journal of Business 64, 1-19.

Detemple, J., Murthy, S., 1994. Intertemporal asset pricing with heterogeneous beliefs. Journal of Economic Theory $62,294-320$.

Duan, J.C., 1995. The GARCH option pricing model. Mathematical Finance 5, 13-32.

Dumas, B.A.J.F., Whaley, R., 1998. Implied volatility functions: empirical tests. Journal of Finance 53, $2059-2106$.

Dumas, B., Kurshev., A., Uppal, R., 2009. Equilibrium portfolio strategies in the prsence of sentiment risk and excess volatility. Journal of Finance 64, 195-229.

Friedman, M., 1953. The case of flexible exchange, in: Essays in Positive Economics Rates. University of Chicago Press, Chicago, IL, USA.

Guidolin, M., Timmermann, A., 2003. Option prices under Bayesian learning: implied volatility dynamics and predictive densities. Journal of Economic Dynamics and Control 27, 717-769.

Guidolin, M., Timmermann, A., 2007. Properties of equilibrium asset prices under alternative learning schemes. Journal of Economic Dynamics and Control 31, 161-217.

Hahn, W., Dyer, J., 2008. Discrete time modelling of mean-reverting stochastic processes for real option valuation. European Journal of Operational Research 184, 534-548.

Heath, D., Platen, E., 2006, A Benchmark Approach to Finance. Springer, Berlin, Germany.

Heston, S., 1993. A closed form solution for options with stocahstic volatility with appllciation to bond and currency options. Review of Financial Studies 6, 327-343.

Heston, S., Nandi, S., 2000. A closed form GARCH option valuation model. Review of Financial Studies 13, 585-625.

Hull, W., White A., 1987. The pricing of options on assets with stochastic volatlities. Journal of Finance 42, 281-300.

Jouini, E., Napp, C., 2006. Heterogeneous beliefs and asset pricing in discrete time: An analysis of pessimism and doubt. Journal of Economic Dynamics and Control 30, 1233-1260.

Jouini, E., Napp, C., 2007. Consensus consumer and intertemporal asset pricing with heterogeneous beliefs. Review of Economic Studies 74, 1149-1174.

Kloeden, P., Platen, E., 1992. Numerical Solution of Stochastic Differential Equations. Springer-Verlag Berlin Heidelberg, Germany.

Kogan, L., Ross, S., Wang, J., Westerfield, M., 2006. The price impact and survival of irrational traders. Journal of Finance 61, 195-229.

Li, T., 2007. Heterogeneous Beliefs, Option Prices, and Volatlity Smiles. SSRN Working Paper. Available online: http://ssrn.com/abstract=890277 (accessed on 18 October 2016).

Melino, A., Turnbull, S., 1990. Pricing foreign currency options with stochastic volatility. Journal of Econometrics 45, 239-265.

Merton, R., 1976. Option pricing when underlying stock returns are discontinuous. Journal of Financial Economics $3,125-144$.

Nelson, D., Ramaswamy, K., 1990. Simple binomial process as diffusion approximations in finanical models. Review of Financial Studies 3, 393-430.

Rubinstein, M., 1985. Nonparametric tests of alternative option pricing models using all reported trades and quotes on the 30 most active CBOE option classes form August 23, 1976 through August 31, 1978. Journal of Finance 40, 455-480. 
Rubinstein, M., 1994. Implied binomial trees. Journal of Finance 49, 771-818. Papers and Proceedings Fifty-Fouth Annual Meeting of the American Finance Association, Boston, MA, USA, January 3-5.

Sandroni, A., 2000. Do markets favor agents able to make accurate predictions? Econometrica 68, $1303-1342$.

Van der Hoek, J., Elliott R., 2006. Binomial Models in Finance. Springer, Berlin, Germany.

Wiggins, J., 1987. Option values under stochastic volatility: Theory and empirical estimates. Journal of Financial Economics 19, 351-372.

Zapatero, F., 1998. Effects of financial innovations on market volatility when beliefs are heterogeneous. Journal of Economic Dynamics and Control 22. 597-626.

(C)2016 by the authors. Published by Science Press (CSPM Ltd.) 